\title{
Change in gastric emptying eight weeks after endo- scopic submucosal dissection in patients with early gastric cancer
}

Authors

Institutions
Ko Watanabe ${ }^{1,2}$, Takuto Hikichi² ${ }^{2}$ Masaki Sato ${ }^{1}$, Jun Nakamura' ${ }^{1}$, Katsutoshi Obara ${ }^{3}$, Hiromasa Ohira ${ }^{1}$

'Department of Gastroenterology and Rheumatology, Fukushima Medical University School of Medicine, Fukushima City, Japan

2 Department of Endoscopy, Fukushima Medical University Hospital, Fukushima City, Japan

3 Department of Advanced Gastroenterological Endoscopy, Fukushima Medical University, Fukushima City, Japan submitted 31. August 2015 accepted after revision 21. February 2016

\section{Bibliography}

Dol http://dx.doi.org/ 10.1055/s-0042-105202 Published online: 21.4.2016 Endoscopy International Open 2016; 04: E597-E602

(c) Georg Thieme Verlag KG Stuttgart . New York E-ISSN 2196-9736

\section{Corresponding author}

\section{Takuto Hikichi, MD, PhD}

Department of Endoscopy

Fukushima Medical University Hospital

1 Hikarigaoka

Fukushima City

Fukushima

Japan 960-1295

Fax: +81-24-547-1586

takuto@fmu.ac.jp
Background: Gastric emptying after endoscopic submucosal dissection (ESD) for early gastric cancer is not clear. The aim of this study was to evaluate changes in gastric emptying from before ESD to 8 weeks after ESD.

Methods: In total, 54 patients with early gastric cancer were enrolled in this study. A breath test with carbon $13\left({ }^{13} \mathrm{C}\right)$ was conducted before ESD and at 1 and 8 weeks after ESD. The $T_{\text {lag }}$ and $T_{1 / 2}$ values were analyzed at each time point. The primary outcomes were the changes in the $T_{\text {lag }}$ and $\mathrm{T}_{1 / 2}$ values from before ESD to 1 and 8 weeks after ESD. The secondary outcomes were the factors associated with the changes in the $T_{\text {lag }}$ and $T_{1 / 2}$ values.

\section{Introduction \\ $\nabla$}

Endoscopic submucosal dissection (ESD), which enables reliable en bloc resection of early gastric cancer, has been developed in Japan and some other countries [1]. ESD is currently a standard treatment for early gastric cancer in Japan, particularly differentiated mucosal cancers with a low risk for lymph node metastasis [2]. Because ESD without nerve resection and lymph node dissection can preserve the normal anatomy of the stomach, various complications related to conventional gastrectomy, including delayed gastric emptying, are avoided. However, some patients experience abdominal distension and loss of appetite [3] and have a large amount of food residue in the stomach even after ESD. Higuchi et al. reported preserved gastric emptying 8 weeks after ESD [4], whereas Uehara et al. reported delayed gastric emptying 6 to 8 days after ESD [3]. Thus, it is controversial whether ESD induces delayed gastric emptying. Moreover, no reports have tracked the change in gastric emptying from before ESD through several weeks after ESD. The aims of this study were to compare gastric empty-
Results: Gastric emptying was delayed at 1 and 8 weeks after ESD compared with before ESD ( $T_{\text {lag }}$ $P=0.002, P<0.001 ; \mathrm{T}_{1 / 2} P=0.005, P=0.001$, respectively). The changes in the $\mathrm{T}_{\text {lag }}$ and $\mathrm{T}_{1 / 2}$ values from before ESD to 1 week after ESD were greater for proximal stomach lesions than for distal stomach lesions $(P=0.028, P<0.001)$. Proximal stomach lesions were identified as the significant factor that influenced changes in the $T_{\text {lag }}$ and $T_{1 / 2}$ values from before ESD to 1 week after ESD in the multivariate analyses ( $\left.\mathrm{T}_{\text {lag }} P=0.003, \mathrm{~T}_{1 / 2} P=0.005\right)$.

Conclusions: ESD induced delayed gastric emptying until 8 weeks after ESD. Proximal stomach lesions were also associated with decreased emptying 1 week after ESD.

ing before ESD with gastric emptying at 1 and 8 weeks after ESD and to identify the factors that influence gastric emptying.

\section{Methods \\ V}

\section{Patients}

In total, 54 patients with early gastric cancer who underwent ESD at Fukushima Medical University Hospital between October 2010 and January 2013 were enrolled in this study. Indications for ESD to treat early gastric cancer included the following: (i) differentiated intramucosal cancer without ulceration; (ii) differentiated intramucosal cancer 3 $\mathrm{cm}$ or less in size with ulceration; and (iii) undifferentiated intramucosal cancer $2 \mathrm{~cm}$ or less in size without ulceration. Other inclusion criteria were age between 20 and 80 years, performance status grade of 0 , and the provision of consent to undergo ESD and a breath test. Patients were excluded from the study if they were younger than 20 or older than 80 years of age; had a performance status grade of 1 or higher; had a previous history of esophageal/gastric surgery or endo- 
scopic treatment; had a severe hepatic, renal, cardiovascular, or respiratory disorder; had dementia; had synchronous multiple lesions of gastric cancer; were unable to stop taking a prokinetic agent or a proton pump inhibitor (PPI) before ESD; or did not agree to undergo ESD. This study was conducted with the approval of the Fukushima Medical University Ethics Committee (approval No.763) and was registered in the University Hospital Medical Information Network (UMIN) as No.UMIN000011523. All of the patients provided written consent to participate in the study.

\section{Endoscopic submucosal dissection}

ESD was performed with a DualKnife (KD-650L; Olympus, Tokyo, Japan) or an IT Knife2 (KD-611L; Olympus). For the submucosal injection, a 1:1 solution of $0.4 \%$ sodium hyaluronate (MucoUp; Johnson \& Johnson K. K., Tokyo, Japan) and glycerol (Chugai Pharmaceutical Co. Ltd., Tokyo, Japan) was injected into the submucosa with a 25-gauge injection needle (Impact Flow; TOP Corp., Tokyo, Japan). Hemostatic forceps (FD410LR Coagrasper; Olympus) were used for the prophylactic coagulation of blood vessels and hemostasis for intraoperative bleeding. The VIO 300 D or ICC 200 (ERBE Elektromedizin, Tübingen, Germany) was used as a highfrequency generator.

\section{Gastric emptying}

A breath test with ${ }^{13} \mathrm{C}$-labeled acetic acid was performed to evaluate gastric emptying before and after ESD. A 200-kcal/200-mL liquid meal (Racol; Otsuka Pharmaceutical Co., Ltd., Tokyo, Japan) was used for the test. Patients fasted overnight for at least 10 hours and underwent the test the following morning. Each patient was instructed to consume a liquid meal containing $100 \mathrm{mg}$ of ${ }^{13} \mathrm{C}$-labeled acetic acid and to exhale into a collection bag at $5,10,15,20,30,40,50,60,75,90,105$, and 120 minutes after ingestion. The concentration of ${ }^{13} \mathrm{CO}_{2}$ in the exhaled breath was measured with an infrared spectrometer (POCone; Otsuka Electronics Co., Ltd., Osaka, Japan). The $\mathrm{T}_{\text {lag }}$ and $\mathrm{T}_{1 / 2}$ values, as proposed by Ghoos et al. [5], were calculated from the ${ }^{13} \mathrm{CO}_{2}$ concentration in the exhaled breath with the Solver function of Excel 2010 (Microsoft; Redmond, Washington, USA) and were used as measures of gastric emptying. The $\mathrm{T}_{\text {lag }}$ value represents the time at which the ${ }^{13} \mathrm{CO}_{2}$ discharge rate reaches the maximum, and the $\mathrm{T}_{1 / 2}$ value represents the time required to discharge $50 \%$ of the total cumulative ${ }^{13} \mathrm{CO}_{2}$ discharge volume. The largest values of $\mathrm{T}_{\text {lag }}$ and $\mathrm{T}_{1 / 2}$ indicate delayed gastric emptying.

\section{Study protocol}

Each patient underwent 3 breath test sessions the day before and at 1 and 8 weeks after ESD. Artificial ulcers after ESD were in the active and healing stages at 1 and 8 weeks after ESD, respectively [6]. All of the prokinetic agents and PPIs were discontinued more than 1 week before ESD. The oral administration of $20 \mathrm{mg}$ of rabeprazole (Pariet; Eisai Co., Ltd., Tokyo, Japan) began on the day of ESD and continued for the subsequent 8 weeks. Oral food intake was suspended from the day of ESD until 3 days after ESD. Endoscopy was performed after ESD to evaluate the grade of artificial ulcer at 1 and 8 weeks after ESD and on the same day as the breath test after breath sampling.

The primary outcomes were changes in the $T_{\text {lag }}$ and $T_{1 / 2}$ values from before ESD to 1 and 8 weeks after ESD. The secondary outcomes were the patients' symptoms at 1 and 8 weeks following ESD, the food residue in the stomach identified by endoscopy at 1 and 8 weeks after ESD, and the factors associated with changes in the $\mathrm{T}_{\text {lag }}$ and $\mathrm{T}_{1 / 2}$ values. The changes in the $\mathrm{T}_{\text {lag }}$ and $\mathrm{T}_{1 / 2}$ values were expressed as the differences between the values before ESD and the values at 1 and 8 weeks after ESD. The lesion location was classified as the "proximal stomach" or the "distal stomach." The proximal stomach was defined as the upper or middle region of the stomach, and the distal stomach was defined as the lower region of the stomach, according to the Japanese Classification of Gastric Carcinoma [7]. The circumferential lesion distribution was classified as "lesser curvature" or "greater curvature." Lesions in the anterior and posterior walls were classified according to whether post-ESD ulcers were located on the lesser or greater curvature. The presence of Helicobacter pylori infection was determined by the serum anti-H. pylori immunoglobulin G (IgG) antibody titer (Otsuka Pharmaceutical Co., Ltd., Tokyo, Japan), and a titer of $10 \mathrm{U} / \mathrm{mL}$ or greater was considered to be a positive result.

\section{Statistical analysis}

The $\mathrm{T}_{\text {lag }}$ and $\mathrm{T}_{1 / 2}$ data are reported as median (interquartile range). Changes in the $T_{\text {lag }}$ and $T_{1 / 2}$ values from before ESD to 1 and 8 weeks after ESD were analyzed with Wilcoxon's signed rank test. The significance of factors associated with changes in $\mathrm{T}_{\text {lag }}$ and $\mathrm{T}_{1 / 2}$ values from before to after ESD was tested with the Mann-Whitney $U$ test, Spearman's rank correlation coefficient $(r s)$, and single and multiple regression analyses. Differences were considered to be statistically significant at a $P$ value below 0.05 . Standardized regression coefficients were reported as $\beta$, and a larger $\beta$ value indicated a greater influence on gastric emptying. Factors that were determined to be significant by single regression analysis, or those that were expected to have a clinical impact, were analyzed by multiple regression analyses. These were performed with IBM SPSS Statistics for Windows, Version 21 (IBM Corp., Armonk, New York, USA).

\section{Results}

$\nabla$

\section{Patient characteristics and outcomes of endoscopic} submucosal dissection

The characteristics of the patients enrolled in the study and the ESD treatment outcomes are summarized in $\bullet$ Table 1. The study population consisted of 34 male and 20 female patients whose mean age was 68.9 years. The mean diameter of the resected specimens was $43.5 \mathrm{~mm}$. Lesions were located in the proximal stomach in 31 patients and in the distal stomach in 23 patients. No lesion examined in this study extended to the esophagogastric junction or the pyloric ring. A total of 32 patients had a positive anti-H. pylori IgG antibody test. Of the 22 patients with a negative antibody test, 3 had undergone successful eradication therapy. However, all of the patients had atrophic gastritis on endoscopy. ESD enabled the en bloc resection of lesions in all of the patients, including 48 patients with mucosal invasion and 6 with submucosal invasion. None of the patients experienced severe complications during or after ESD. According to the Sakita-Miwa classification [8], the status of the post-ESD ulcers at 8 weeks following ESD was "scarring stage" in 36 patients and "healing stage" in 18 patients. There were 9 patients $(16.7 \%)$ who experienced mild abdominal distension or mild abdominal discomfort 1 week after ESD. Only 1 patient (1.9\%) reported mild abdominal distension 8 weeks after ESD. Food residues in the stomach were found in 4 patients (7.4\%) at endoscopy conducted 1 week after ESD. Food residues were observed in 2 patients $(3.7 \%) 8$ weeks after ESD. 
Table 1 Clinical characteristics of patients and outcomes of endoscopic mucosal dissection (ESD).

\begin{tabular}{|lc|}
\hline Age, mean \pm SD, $y$ & $68.9 \pm 6.6$ \\
\hline Male/female patients, $\mathrm{n}$ & $34 / 20$ \\
\hline Height, mean \pm SD, cm & $159.1 \pm 9.5$ \\
\hline Weight, mean \pm SD, $\mathrm{kg}$ & $60.3 \pm 12.5$ \\
\hline Body mass index, mean \pm SD & $23.8 \pm 4.2$ \\
\hline History of gastric ulcer (absent/present), $\mathrm{n}$ & $51 / 3$ \\
\hline History of duodenal ulcer (absent/present), & $54 / 0$ \\
\hline History of diabetes mellitus (absent/present), $\mathrm{n}$ & $47 / 7$ \\
\hline Diameter of resected specimens, mean \pm SD, mm & $43.5 \pm 16.5$ \\
\hline Size of resected specimens, mean \pm SD, mm ${ }^{2}$ & $1603.9 \pm 1237.9$ \\
\hline Lesion location (proximal/distal), n & $31 / 23$ \\
\hline Circumferential lesion distribution (lesser/greater), & \\
\hline ncurvature) cccc curvat) curve) & $40 / 14$ \\
\hline Depth of tumor invasion (mucosal/submucosal), $\mathrm{n}$ & $48 / 6$ \\
\hline Ulcer finding under tumor (absent/present), n & $49 / 5$ \\
\hline Procedure duration, mean \pm SD, min & $92.1 \pm 76.1$ \\
\hline Anti-H. pylori lgG antibodies (positive/negative), n & \\
\hline (eradicated)) & $32 / 22\left(3^{*}\right)$ \\
\hline Atrophic gastritis (present/absent), n & $54 / 0$ \\
\hline State of post-ESD ulcer 8 weeks after ESD (S/H), n & $36 / 18$ \\
\hline
\end{tabular}

SD, standard deviation; H. pylori, Helicobacter pylori; IgG, immunoglobulin G; $\mathrm{S}$, scarring stage; $\mathrm{H}$, healing stage.

* Patients with successful eradication therapy.

\section{Gastric emptying}

The median $\mathrm{T}_{\mathrm{lag}}$ values before, at 1 week after, and at 8 weeks after ESD were 1.03 (interquartile range [IQR] $0.92-1.20$ ), 1.09 (IQR 1.00-1.26), and 1.10 (IQR 1.01-1.32) hours, respectively, which indicated delayed emptying at 1 and 8 weeks after ESD compared with emptying before ESD $(P=0.002$ and $P<0.001$, respectively). No significant difference was observed between the 1- and 8-week post-ESD $\mathrm{T}_{\operatorname{lag}}$ values ( 0 Fig. $1 \mathrm{a}$ ). The corresponding median $\mathrm{T}_{1 / 2}$ values were 1.58 (IQR $1.43-1.82$ ), 1.73 (IQR 1.50-2.11), and 1.75 (IQR $1.58-2.03$ ) hours, respectively, which again indicated delayed emptying at 1 and 8 weeks after ESD compared with emptying before ESD $(P=0.005$ and $P=$ 0.001 , respectively), and there was no significant difference between the $T_{1 / 2}$ values at 1 and 8 weeks after ESD ( $\bullet$ Fig. $\mathbf{1}$ b).

Age, gender, body height and weight, body mass index, medical history of diabetes mellitus, history of gastric ulcer, procedure duration, depth of tumor invasion, circumferential lesion distribution, and the presence of an ulcer under the tumor were not correlated with the changes in the $T_{\operatorname{lag}}$ and $T_{1 / 2}$ values at 1 and 8 weeks after ESD compared with those before ESD, whereas the diameter of the resected specimen was weakly correlated with the changes in the $\mathrm{T}_{1 / 2}$ value $(r s=0.29, P=0.04)$ but not in the $\mathrm{T}_{\mathrm{lag}}$ value at 1 week after ESD compared with the values before ESD. No correlation was observed between the diameter of the resected specimen and the change in gastric emptying from before to 8 weeks after ESD. The state of post-ESD ulcer was not correlated with the change in either $T_{\text {lag }}$ or $T_{1 / 2}$ at 8 weeks after ESD. Proximal stomach lesions were associated with greater changes in $\mathrm{T}_{\text {lag }}$ and $\mathrm{T}_{1 / 2}$ at 1 week after ESD compared with before ESD, which indicated a greater decrease in gastric emptying $(P=0.028$ and $P<$ 0.001 , respectively) ( $\bullet$ Fig. 2 ), whereas no significant difference was observed between the proximal and distal stomach lesions with respect to changes in gastric emptying before ESD and at 8 weeks after ESD ( $\bullet$ Fig. 3 ). The presence of symptoms and food residues in the stomach after ESD were not correlated with the changes in the $T_{\text {lag }}$ and $T_{1 / 2}$ values at 1 and 8 weeks after ESD compared with those before ESD.
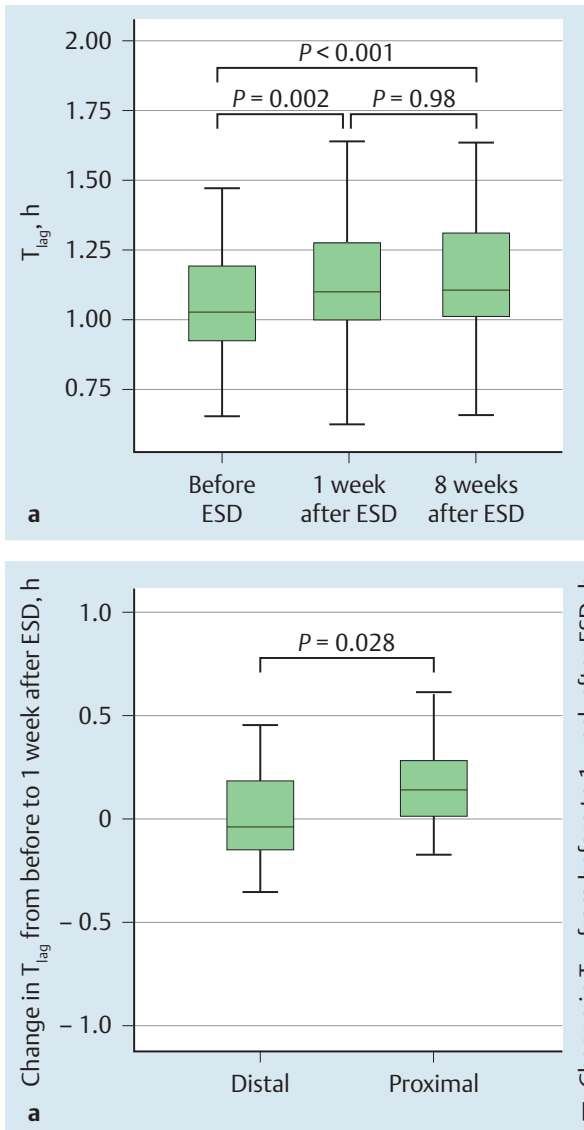
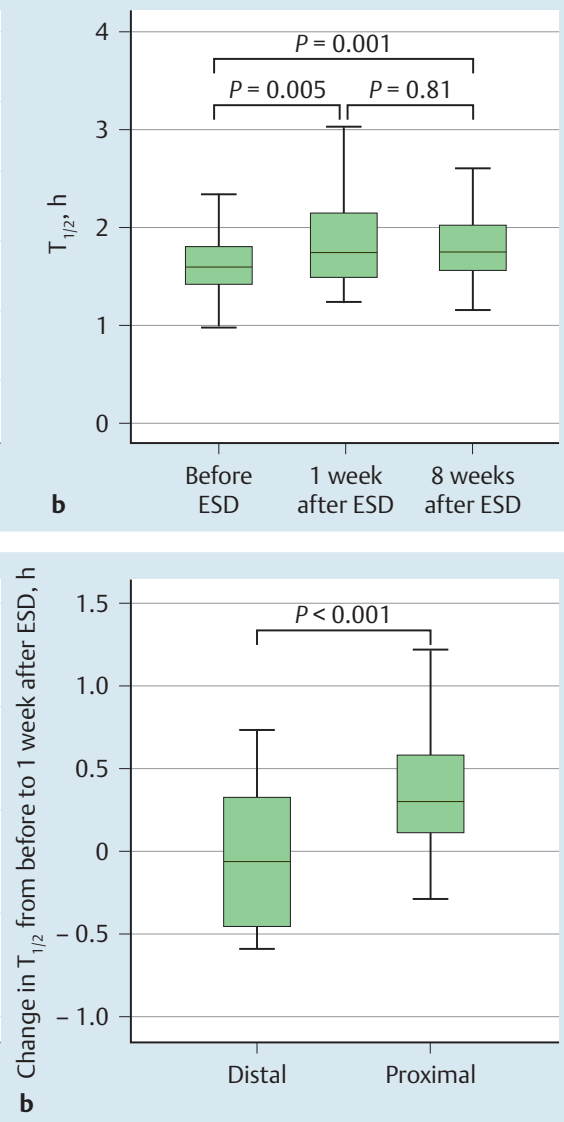

Fig. 1 Changes in the $T_{\text {lag }}(\mathbf{a})$ and $T_{1 / 2}$ (b) values from before endoscopic submucosal dissection (ESD) to 1 and 8 weeks after ESD in 54 patients being treated for early gastric cancer. Delayed $\mathrm{T}_{\text {lag }}$ and $T_{1 / 2}$ values were noted at 1 and 8 weeks after ESD compared with the pre-ESD values, with no significant difference between the 1- and 8-week post-ESD values (Wilcoxon's signed rank test).

Fig.2 Relationship between changes in the $\mathrm{T}_{\text {lag }}$ (a) and $T_{1 / 2}$ (b) values from before endoscopic submucosal dissection (ESD) to 1 week after ESD and the lesion locations. Proximal stomach lesions were associated with greater changes in the $\mathrm{T}_{\text {lag }}$ and $\mathrm{T}_{1 / 2}$ values from before ESD to 1 week after ESD compared with distal stomach lesions (Mann-Whitney $U$ test). 

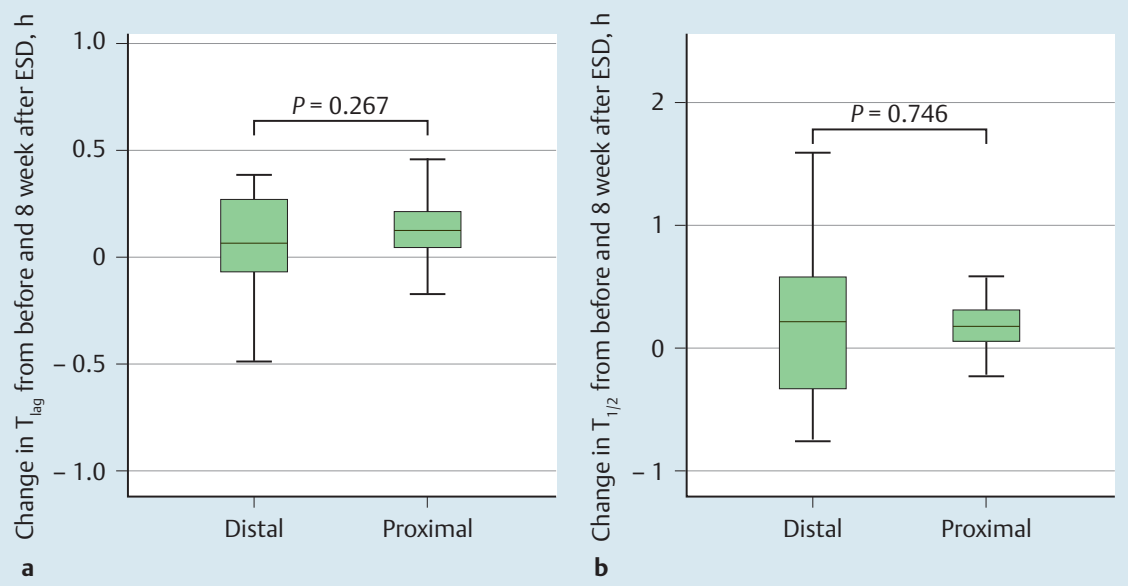

Fig. 3 Relationship between changes in the $\mathrm{T}_{\text {lag }}$ (a) and $\mathrm{T}_{1 / 2}$ (b) values from before endoscopic submucosal dissection (ESD) to 8 weeks after ESD and the lesion locations. No significant differences were observed between proximal and distal stomach lesions with respect to changes in the $T_{\text {lag }}$ and $T_{1 / 2}$ values from before ESD to 8 weeks after ESD (MannWhitney $U$ test).

In the single regression analysis, proximal stomach lesions were identified as the only significant factor influencing the changes in the $\mathrm{T}_{\text {lag }}$ and $\mathrm{T}_{1 / 2}$ values from before to 1 week after $\operatorname{ESD}(P=0.018$ and $P=0.003$, respectively) ( Table 2 ). Multiple regression analysis also identified proximal stomach lesions as the only significant factor influencing the changes in $\mathrm{T}_{\text {lag }}$ and $\mathrm{T}_{1 / 2}$ values from before to 1 week after $\operatorname{ESD}(P=0.033$ and $P=0.005$, respectively) ( $\bullet$ Table 2). No factor was identified as influencing the changes in $\mathrm{T}_{\text {lag }}$ and $\mathrm{T}_{1 / 2}$ values from before to 8 weeks after ESD based on either single or multiple regression analysis ( $\bullet$ Table 3 ).

\section{Discussion}

This study found that delayed gastric emptying occurred 1 week after ESD and continued until 8 weeks after ESD. In addition, proximal stomach lesions were identified as being associated with delayed gastric emptying 1 week after ESD.

Scintigraphy has traditionally been considered the gold standard for evaluating gastric emptying [9]. However, scintigraphy has been associated with problems such as exposure to radiation, high cost, and lengthy test time. In contrast, the $\mathrm{T}_{\text {lag }}$ and $\mathrm{T}_{1 / 2}$ values can be easily and repeatedly measured with a ${ }^{13} \mathrm{C}$ breath test in a minimally invasive technique, and the results correlate with scintigraphy findings $[5,9]$. We therefore used this method to evaluate gastric emptying in our study.

Gastric emptying is reduced in patients with gastric ulcers [1012]. As in our study, delayed gastric emptying after endoscopic treatment was reported by Kawamura et al. at 4 days after endoscopic mucosal resection [13] and by Uehara et al. at 6 to 8 days after ESD [3]. Higuchi et al. reported preserved gastric emptying at 8 weeks after ESD [4]. Therefore, at 8 weeks after ESD, the results of the current study were different from those of the study reported by Higuchi et al. The reason for this difference could be explained by the smaller sample size and smaller size of the resected specimens in their study compared with the current study.

Delayed mobility in gastrointestinal disorders has been associated with reduced contraction of inflamed smooth muscles, which is caused by Th1 and Th2 cytokines [14]. Thus, the delayed gastric emptying 1 week after ESD was likely due to inflammatory changes induced by artificial ulceration. Additionally, previous

Table 2 Single regression analysis and multiple regression analysis of factors associated with the changes in $\mathrm{T}_{\text {lag }}$ and $\mathrm{T}_{1 / 2}$ from before to 1 week after endoscopic submucosal dissection.

\begin{tabular}{|c|c|c|c|c|c|c|c|c|}
\hline & \multicolumn{4}{|c|}{ Single regression analysis } & \multicolumn{4}{|c|}{ Multiple regression analysis } \\
\hline & \multicolumn{2}{|l|}{$\mathbf{T}_{\text {lag }}$} & \multicolumn{2}{|l|}{$T_{1 / 2}$} & \multicolumn{2}{|l|}{$\mathbf{T}_{\text {lag }}$} & \multicolumn{2}{|l|}{$T_{1 / 2}$} \\
\hline & $\boldsymbol{\beta}$ & $P$ value & $\beta$ & $P$ value & $\beta$ & $P$ value & $\boldsymbol{\beta}$ & $P$ value \\
\hline Age & -0.271 & 0.844 & 0.111 & 0.423 & - & - & - & - \\
\hline Gender & -0.102 & 0.966 & -0.004 & 0.978 & - & - & - & - \\
\hline Height & 0.059 & 0.674 & -0.026 & 0.852 & - & - & - & - \\
\hline Weight & 0.111 & 0.426 & 0.078 & 0.574 & - & - & - & - \\
\hline Body mass index & 0.166 & 0.231 & 0.107 & 0.440 & - & - & - & - \\
\hline History of diabetes mellitus & 0.010 & 0.942 & -0.005 & 0.972 & - & - & - & - \\
\hline History of gastric ulcer & 0.126 & 0.364 & 0.070 & 0.616 & - & - & - & - \\
\hline Diameter of the resected specimens & 0.157 & 0.257 & 0.121 & 0.382 & 0.133 & 0.509 & 0.112 & 0.570 \\
\hline Size of the resected specimens & 0.171 & 0.216 & 0.150 & 0.280 & - & - & - & - \\
\hline Lesion location & 0.321 & 0.018 & 0.392 & 0.003 & 0.312 & 0.033 & 0.403 & 0.005 \\
\hline Circumferential lesion distribution & 0.017 & 0.905 & -0.065 & 0.639 & 0.063 & 0.650 & -0.029 & 0.831 \\
\hline Depth of tumor invasion & -0.101 & 0.467 & 0.012 & 0.934 & - & - & - & - \\
\hline Ulcer under the tumor & 0.145 & 0.295 & 0.117 & 0.401 & - & - & - & - \\
\hline Procedure duration & 0.138 & 0.318 & 0.095 & 0.496 & -0.051 & 0.805 & -0.124 & 0.536 \\
\hline Symptoms & 0.073 & 0.600 & -0.045 & 0.746 & - & - & - & - \\
\hline Food residues & 0.074 & 0.595 & 0.112 & 0.421 & - & - & - & - \\
\hline
\end{tabular}


Table 3 Single regression analysis and multiple regression analysis of factors associated with the changes in $T_{\text {lag }}$ and $T_{1 / 2}$ from before to 8 weeks after endoscopic submucosal dissection.

\begin{tabular}{|c|c|c|c|c|c|c|c|c|}
\hline & \multicolumn{4}{|c|}{ Single regression analysis } & \multicolumn{4}{|c|}{ Multiple regression analysis } \\
\hline & $\mathbf{T}_{\text {lag }}$ & & $T_{1 / 2}$ & & $\mathbf{T}_{\text {lag }}$ & & $T_{1 / 2}$ & \\
\hline & $\beta$ & $P$ value & $\beta$ & $P$ value & $\beta$ & $P$ value & $\beta$ & $P$ value \\
\hline Age & -0.135 & 0.329 & -0.087 & 0.533 & - & - & - & - \\
\hline Gender & 0.006 & 0.966 & 0.040 & 0.776 & - & - & - & - \\
\hline Height & 0.012 & 0.933 & 0.050 & 0.719 & - & - & - & - \\
\hline Weight & -0.059 & 0.705 & 0.061 & 0.661 & - & - & - & - \\
\hline Body mass index & -0.008 & 0.624 & 0.041 & 0.766 & - & - & - & - \\
\hline History of diabetes mellitus & 0.097 & 0.486 & 0.666 & 0.060 & - & - & - & - \\
\hline History of gastric ulcer & 0.035 & 0.802 & -0.007 & 0.961 & - & - & - & - \\
\hline Diameter of the resected specimens & 0.057 & 0.680 & -0.072 & 0.606 & -0.021 & 0.919 & -0.134 & 0.517 \\
\hline Size of the resected specimens & 0.085 & 0.539 & -0.039 & 0.780 & - & - & - & - \\
\hline Lesion location & 0.816 & 0.179 & 0.189 & 0.780 & 0.175 & 0.243 & 0.217 & 0.142 \\
\hline Circumferential lesion distribution & -0.055 & 0.692 & -0.148 & 0.286 & -0.037 & 0.798 & -0.162 & 0.255 \\
\hline Depth of tumor invasion & -0.006 & 0.963 & 0.014 & 0.923 & - & - & - & - \\
\hline Ulcer finding under the tumor & 0.181 & 0.191 & 0.123 & 0.375 & - & - & - & - \\
\hline Procedure duration & 0.088 & 0.527 & -0.030 & 0.827 & 0.040 & 0.853 & -0.031 & 0.882 \\
\hline State of post-ESD ulcer & 0.055 & 0.695 & -0.71 & 0.611 & - & - & - & - \\
\hline Symptoms & -0.004 & 0.975 & 0.035 & 0.800 & - & - & - & - \\
\hline Food residues & -0.082 & 0.556 & 0.010 & 0.944 & - & - & - & - \\
\hline
\end{tabular}

studies have reported that ghrelin accelerates gastric emptying $[15,16]$. Ghrelin is a gastrointestinal hormone secreted mainly from the gastric mucosa. Therefore, the delayed gastric emptying 1 week after ESD was likely due to inflammatory changes induced by artificial ulceration and decreased ghrelin secretion resulting from mucosal resection.

The lesion location was associated with delayed gastric emptying 1 week after ESD, with a greater decrease noted after ESD for lesions in the proximal stomach than for those in the distal stomach. The most likely reason for this result may be the difference in emptying function between the proximal and distal stomach. The proximal stomach relaxes in an isobaric fashion to store food and then gradually increases the inner pressure by increasing the wall tension to push food to the distal stomach. Conversely, the distal stomach mixes gastric acid and food mass via periodic peristalsis to promote digestion, and then it breaks down solid food into 1- to 2-mm pieces and pushes them out through the pylorus [17]. However, controversy remains regarding the association between peptic ulcer location and the degree of alteration in emptying function $[10,18-21]$.

It is not appropriate to conclude that post-ESD ulcers that form in the proximal stomach are more likely to affect gastric emptying because of the functional difference between the proximal and distal stomach. Another possible explanation is the difference in wall thickness between the proximal and distal stomach. Excessive electrocauterization during ESD can cause delayed perforation [22]. Similarly, a post-ESD artificial ulcer, unlike a peptic ulcer, is accompanied by smooth muscle injury caused by electrocauterization. The submucosa and muscularis propria are thinner in the proximal stomach than in the distal stomach, and the proximal stomach is thus more vulnerable to injury and a subsequent decrease in emptying function. Another unclarified issue is the reason for the sustained decrease in gastric emptying that lasted until 8 weeks after ESD, when the ulcer was thought to be in the healing stage. Although one possible explanation might be the sustained damage to the smooth muscle caused by ESD, the absence of a location-related difference in decreased emptying 8 weeks after ESD suggests a mechanism altogether different from the functional decrease noted 1 week after ESD.

Although gastric emptying in patients with gastric ulcers improves as the healing process proceeds $[18,19]$, some reports have suggested that the emptying function of patients with recurrent gastric ulcers remains decreased even during the healing stage $[23,24]$. A post-ESD ulcer covers a relatively large area and can result in severe fibrosis and morphological changes in the stomach wall. Such changes, similar to those observed in patients with recurrent gastric ulcers, may have been responsible for the sustained decrease in emptying function that lasted until 8 weeks after ESD. In the current study, $16.7 \%$ of the patients experienced mild abdominal distension or discomfort 1 week after ESD. Nevertheless, delayed gastric emptying continued until 8 weeks after ESD. The proportion of patients who reported abdominal distension decreased to $1.9 \%$ by 8 weeks after ESD. Furthermore, symptoms and food residues in the stomach after ESD were not correlated with the changes in gastric emptying in this study. Delayed gastric emptying after ESD might be latent in almost all cases. Moreover, the abdominal symptoms after ESD might be associated with multiple factors, such as abdominal pain associated with a post-ESD artificial ulcer, physiological factors, and gastric emptying. Therefore, the symptoms might decrease over time, unlike the delay in gastric emptying. Metachronous recurrences after ESD have recently become an important difficulty [25]. Because repeated ESD procedures for metachronous tumors might cause more severe fibrosis and a greater number of morphological changes in the stomach wall, delayed gastric emptying after repeated ESD procedures for metachronous tumors might induce a greater delay in gastric emptying and more severe symptoms than those of the patients examined in the present study. Prokinetic agents might be effective for patients who have symptoms or undergo repeated ESD procedures. Additional studies must be conducted to elucidate these points. PPIs are effective in preventing bleeding after ESD [26] and thus were orally administered to the patients in this study from the day of ESD until 8 weeks after the procedure. Some investigators 
have reported that PPIs do not affect the gastric emptying function $[17,27,28]$, whereas others have reported that these drugs negatively affect the emptying function $[17,29,30]$. The use of PPIs may have been responsible for the decreased gastric emptying observed in the present study. However, it should be noted that all of the patients had underlying atrophic gastritis and likely had reduced gastric acid secretion at baseline. Therefore, the patients were likely to be less responsive to the effect of PPIs.

This study has several limitations. First, a liquid meal was used for the test. Unlike a liquid, solid food must be broken down into pieces $2 \mathrm{~mm}$ or smaller in the gastric antrum [17], and the discharge from solid food is more influenced by antral function. Therefore, future studies should use solid food in a similar study procedure because doing so might yield different results regarding the association between lesion location and the degree of decrease in emptying function. Second, the observation period was 8 weeks after ESD, which corresponds to the healing stage of an ulcer. Further investigations are needed to determine the changes in gastric emptying over a longer period of time. Finally, the precise mechanisms of delayed gastric emptying remain unclear. Additional studies are needed to prove physiological or histological mechanisms.

In conclusion, the present study is significant because it demonstrates delayed gastric emptying at both 1 and 8 weeks after gastric ESD. Proximal stomach lesions were identified as being associated with delayed gastric emptying 1 week after ESD. Future studies should examine changes in gastric emptying function following ESD over a longer period of time.

\section{Competing interests: None}

\section{Acknowledgments \\ $\nabla$}

We express our gratitude to Tadayuki Takagi, Rei Suzuki, Mitsuru Sugimoto, Naoki Konno, Hitomi Kikuchi, Yuichi Waragai, Hiroyuki Asama, Mika Takasumi, and all of the endoscopy medical staff in Fukushima Medical University Hospital for their collaboration and assistance with the endoscopic procedures and breath tests. We also thank Aya Goto (Department of Public Health, Fukushima Medical University School of Medicine) for assisting us with the statistical analysis.

\section{References}

1 Oda I, Saito D, Tada $M$ et al. A multicenter retrospective study of endoscopic resection for early gastric cancer. Gastric Cancer 2006; 9: 262 270

2 Isomoto H, Shikuwa S, Yamaguchi N et al. Endoscopic submucosal dissection for early gastric cancer: a large-scale feasibility study. Gut 2009; 58: 331 - 336

3 Uehara R, Isomoto H, Minami $\mathrm{H}$ et al. Characteristics of gastrointestinal symptoms and function following endoscopic submucosal dissection and treatment of the gastrointestinal symptoms using rikkunshito. Exp Ther Med 2013; 6: 1083-1088

4 Higuchi $N$, Nakamura K, Ihara $E$ et al. Preserved gastric motility in patients with early gastric cancer after endoscopic submucosal dissection. J Gastroenterol Hepatol 2013; 28: 494-498

5 Ghoos YF, Maes BD, Geypens BJ et al. Measurement of gastric emptying rate of solids by means of a carbon-labeled octanoic acid breath test. Gastroenterology 1993; 104: 1640-1647
6 Kakushima N, Yahagi N, Fujishiro M et al. The healing process of gastric artificial ulcers after endoscopic submucosal dissection. Dig Endosc 2004; 16: 327-331

7 Japanese Gastric Cancer Association. Japanese classification of gastric carcinoma: 3rd English edition. Gastric Cancer 2011; 14: 101 - 112

8 Sakita T, Oguro Y, Takasu S et al. Observations on the healing of ulcerations in early gastric cancer. The life cycle of the malignant ulcer. Gastroenterology 1971; 60: 835-844

9 Parkman HP, Jones MP. Tests of gastric neuromuscular function. Gastroenterology 2009; 136: 1526-1543

10 Griffith GH, Owen GM, Campbell $H$ et al. Gastric emptying in health and in gastroduodenal disease. Gastroenterology 1968; 54: 1-7

11 Miller LJ, Malagelada JR, Longstreth GF et al. Dysfunctions of the stomach with gastric ulceration. Dig Dis Sci 1980; 25: 857-864

12 Stanghellini V, Ghidini C, Maccarini MR. Fasting and postprandial gastrointestinal motility in ulcer and non-ulcer dyspepsia. Gut 1992; 33: $184-190$

13 Kawamura O, Kusano M, Shimoyama Yet al. Oral erythromycin accelerates impaired gastrointestinal motility after endoscopic mucosal resection. Dig Dis Sci 2007; 52: 2211 -2220

14 Akiho H, Ihara E, Motomura $Y$ et al. Cytokine-induced alterations of gastrointestinal motility in gastrointestinal disorders. World J Gastrointest Pathophysiol 2011; 2: 72-81

15 Tack J, Depoortere I, Bisschops R et al. Influence of ghrelin on interdigestive gastrointestinal motility in humans. Gut 2006; 55: 327-333

16 Levin F, Edholm T, Schmidt PT et al. Ghrelin stimulates gastric emptying and hunger in normal-weight humans. J Clin Endocrinol Metab 2006; 91: $3296-3302$

17 Sanaka M, Yamamoto T, Kuyama Y. Effects of proton pump inhibitors on gastric emptying: a systematic review. Dig Dis Sci 2010; 55: $2431-2440$

18 Kanaizumi T, Nakano H, Matsui T et al. Gastric emptying in patients with gastric and duodenal ulcer. Tohoku J Exp Med 1989; 158: 133 140

19 Harasawa S, Tani N, Suzuki S et al. Gastric emptying in normal subjects and patients with peptic ulcer: a study using the acetaminophen method. Gastroenterol Jpn 1979; 14: 1 - 10

20 Miyaoka T, Misaki F, Sasaki Z et al. Dynamic aspects of gastric emptying in patients with peptic ulcer according to ulcer stages. Digestion 1977; 16: $10-17$

21 Garrett JM, Summerskill WH, Code CF. Antral motility in patients with gastric ulcer. Am J Dig Dis 1966; 11: 780 - 789

22 Hanaoka $N$, Uedo $N$, Ishihara $R$ et al. Clinical features and outcomes of delayed perforation after endoscopic submucosal dissection for early gastric cancer. Endoscopy 2010; 42: 1112-1115

23 Kamiya T, Kobayashi Y, Hirako M et al. Gastric motility in patients with recurrent gastric ulcers. J Smooth Muscle Res 2002; 38: 1 -9

24 Kamiya T, Kobayashi Y, Misu $N$ et al. Gastric myoelectrical activity in patients with recurrent gastric or duodenal ulcers. J Smooth Muscle Res 2003; 39: 1-10

25 Kato M, Nishida T, Yamamoto $K$ et al. Scheduled endoscopic surveillance controls secondary cancer after curative endoscopic resection for early gastric cancer: a multicentre retrospective cohort study by Osaka University ESD study group. Gut 2013; 62: 1425-1432

26 Uedo N, Takeuchi Y, Yamada T et al. Effect of a proton pump inhibitor or an H2-receptor antagonist on prevention of bleeding from ulcer after endoscopic submucosal dissection of early gastric cancer: a prospective randomized controlled trial. Am J Gastroenterol 2007; 102: $1610-1616$

27 Takahashi Y, Amano Y, Yuki T et al. Influence of acid suppressants on gastric emptying: cross-over analysis in healthy volunteers. J Gastroenterol Hepatol 2006; 21: 1664-1668

28 Jones MP, Shah D, Ebert CC. Effects of rabeprazole sodium on gastric emptying, electrogastrography, and fullness. Dig Dis Sci 2003; 48: $69-73$

29 Terai S, Iijima K, Asanuma K et al. Lack of modulation of gastric emptying by dietary nitrate in healthy volunteers. Tohoku J Exp Med 2009; 218: $73-79$

30 Sanaka M, Yamamoto T, Kuyama Y. Does rabeprazole enhance distension-induced gastric accommodation? Dig Dis Sci 2009; 54: 416-418 